\title{
Malignant Duodenal Obstruction Caused by Urothelial Carcinoma
}

\author{
Dylan Johnson ${ }^{1 *}$, Ankita Mishra ${ }^{1}$, Kai Huang', Chukwuyem Obia ${ }^{2}$, Subhasis Misra ${ }^{1}$ \\ Department of General Surgery, USF Morsani College of Medicine Graduate Medical Education, USA \\ Department of Pathology, USF Morsani College of Medicine Graduate Medical Education, USA
}

*Corresponding author: Dylan Johnson, MD, HCA Brandon Regional Hospital/USF Morsani College of Medicine Graduate Medical Education, Department of General Surgery, Brandon, FL 33511, USA.

To Cite This Article: Dylan Johnson. Malignant Duodenal Obstruction Caused by Urothelial Carcinoma. 2020 - 9(6). AJBSR.MS.ID.001441. DOI: 10.34297/AJBSR.2020.09.001441.

Received: 眥June 30, 2020; Published: 橎 July 31, 2020

\begin{abstract}
A 79-year-old male presented with several weeks of abdominal pain associated with nausea and vomiting. He was found to have an obstruction of the duodenum and hepatobiliary system that was highly suggestive of malignancy. A thorough evaluation revealed no indication of metastatic disease. The patient elected to undergo a palliative, if not potentially curative, Whipple procedure. After a successful operation, the final report from pathology was highly unexcepted. The patient's duodenal obstruction had been caused by metastatic urothelial carcinoma within the pancreas. Metastatic disease to the pancreas is an exceptionally rare occurrence, more so when considering occult primary tumors of urogenital origin. This is the first description of a patient presenting with duodenal obstruction caused by urothelial carcinoma metastasizing to the pancreas. The following case highlights the aggressive nature of this disease while offering insights into the management and outcomes that were involved in this rare clinical scenario.

Key words: Malignant; Duodenal; Obstruction; Urothelial; Carcinoma; Bladder; Cancer

Abbreviations: UC: Urothelial Carcinoma; ED: Emergency Department; TURP: Transuretheral resection of the prostate; CT: Computated Tomography; EGD: Esophagogastroduodenoscopy; NGT; Nasogastric tube; PET: Positron Emission Tomography; GJ: Gastrojejunal; UGT: Urogenital tract; IHC: Immunohistochemistry; TURB: Transurethral resection of the bladder; RC: Radical Cystectomy
\end{abstract}

\section{Introduction}

The vast majority of pathologic findings after pancreaticoduodenectomy are malignant, the most common of which include pancreatic adenocarcinoma and cholangiocarcinoma [1]. The percentage of metastatic disease to the pancreas discovered after a Whipple procedure is a less than $1 \%$ [2]. Of this $1 \%$, the most common metastases include renal cell (48\%), lung (21\%), colon (14\%), melanoma (9\%), and breast (8\%) [3].

Urothelial carcinoma (UC), also referred to as transitional cell carcinoma, is a particularly aggressive urogenital malignancy capable of widespread metastasis [4]. The most common sites of metastasis are lymph nodes, bone, lungs, and liver [5,6]. Although uncommon, metastases have been described at a number of other locations including the stomach, duodenum, skin, muscle and eyes [7-11]. Here, we describe the case of a patient who underwent a Whipple procedure after presenting with duodenal obstruction secondary to a periampullary mass that turned out to be UC metastasizing to the head of the pancreas.

\section{Case Report}

A 79 year old man presented to the emergency department (ED) for abdominal pain of several weeks' duration associated with unrelenting nausea and vomiting. He had lost approximately 10 lbs during this time which he attributed to poor nutritional 
intake. His medical history was significant for kidney stones and benign prostatic hyperplasia. His surgical history included several ureteroscopies for stone extractions and transurethral resection of the prostate (TURP). He had been adherent with all age-appropriate screening and had no history of cancer otherwise. He did not drink alcohol and had no history of smoking. He had no familial history of cancer. Of note, he had recently seen his primary care physician for episodic hematuria. The urine analysis ordered by his doctor was normal, but the computated tomographic (CT) imaging obtained was notable for a small lower ureteral stone and hydronephrosis.

On initial exam, he was afebrile and hemodynamically stable. He was grossly jaundiced. His abdomen markedly distended but nontender. There were no appreciable massesor lymphadenopathy. Laboratory studies were notable for cholestatic transaminitis along with elevated lipase [AST 306 U/L, ALT 592 U/L, total bilirubin
$2.9 \mu \mathrm{mol} / \mathrm{L}$, direct bilirubin $2.3 \mu \mathrm{mol} / \mathrm{L}$, alkaline phosphatase 296 U/L, lipase $603 \mathrm{U} / \mathrm{L}$, and INR 0.9]. Besides acute kidney injury, the rest of his labs were largely unremarkable [serum creatinine $1.3 \mu \mathrm{mol} / \mathrm{L}$, BUN 21mmol/L, white blood cell count $9.0 \times 109 / \mathrm{L}$, hemoglobin $15.5 \mathrm{mmol} / \mathrm{L}$, platelets 197 x 109/L, and calcium 9.9 $\mathrm{mmol} / \mathrm{L}]$. His albumin and pre-albumin were $3.8 \mathrm{~g} / \mathrm{dL}$ and $14.6 \mathrm{mg} /$ $\mathrm{dL}$, respectively. Urine analysis showed moderate red blood cells, consistent with his history of a recent ureteral stone. CT imaging revealed dilatation of the stomach, proximal duodenum, and biliary tree with normal pancreatic anatomy (Figure 1). Additional findings included right side hydronephrosis with dilatation of the proximal half of the right ureter as well as a bladder floor irregularity and moderately enlarged prostate (Figure 2). Urological consultation advised that these findings were consistent with the patient's history of TURP, ureteroscopy and recent nephrolithiasis.
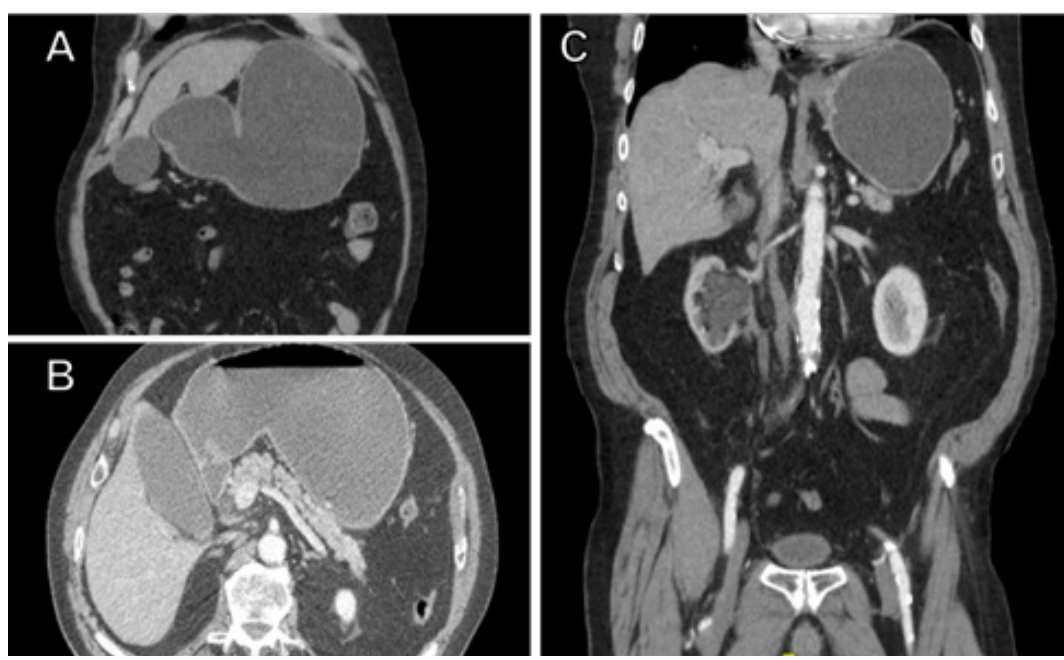

Figure 1: A) CT (coronal view) displaying gastric dilatation. B) CT (axial view) displaying gastric and duodenal dilatation with normal pancreatic anatomy. C) СT (coronal view) displaying gastric dilatation and right side hydronephrosis with ureteral dilatation.

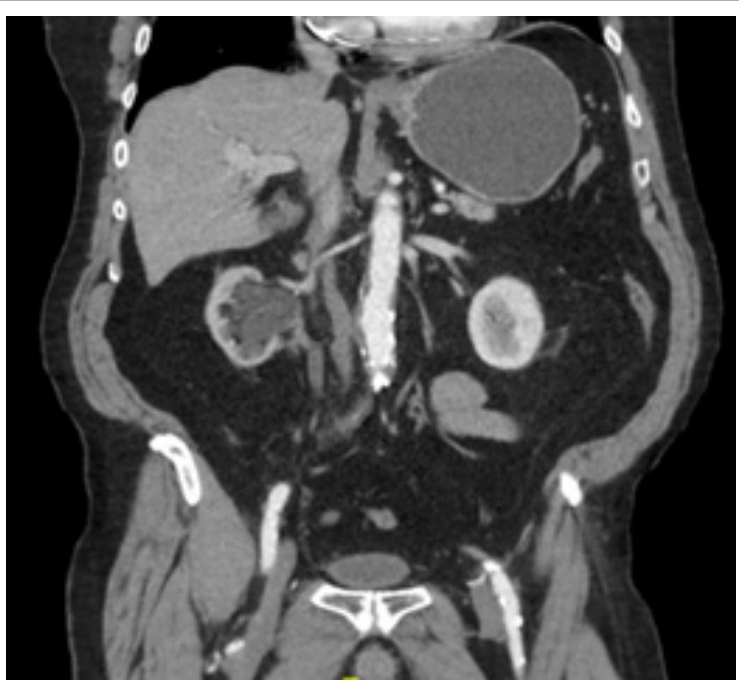

Figure 2: CT (coronal view) displaying gastric dilatation and right side hydronephrosis with ureteral dilatation. 
Management proceeded with esophagogastroduodenoscopy (EGD) which revealed high-grade obstruction in the 2nd and 3rd portions of the duodenum with stenosis and ulceration. A biopsy was taken at that time but later returned negative for malignancy. Following EGD, serum markers were obtained and notable for an elevated CA19-9 [CA19-9 60.9, alpha-fetoprotein 3.0 and carcinoembryonic antigen 1.2.]. A staging workup was completed and showed no evidence of metastatic disease.

While undergoing diagnostic testing, the patient was treated with nasogastric tube (NGT) decompression and started on total parenteral nutrition shortly thereafter. Given the strong suspicion for periampullary malignancy, the patient was counseled regarding the available treatment options, including a potentially definitive Whipple procedure, gastrojejunostomy bypass, or additional endoscopic biopsies under ultrasound guidance. After a lengthy discussion with the patient and his family, they elected to undergo surgical resection of the mass.
In the operating room, gallbladder distension was noted in conjunction with a palpable mass in the descending portion of the duodenum. Additionally, there was severe desmoplastic reaction at the 3rd portion of the duodenum adhering it to the superior mesenteric vein and colonic mesentery. There was no evidence of intraperitoneal metastatic disease. With these findings, the surgical team proceeded with pancreaticoduodenectomy. Intraoperative frozen sections confirmed invasive carcinoma in the head of the pancreas with negative margins at the superior mesenteric vein as well as the bile and pancreatic ducts (Figure 3). Final pathology later returned as an $\mathrm{R} 0$ resection of carcinoma with extensive angiolymphatic invasion and involvement of pancreatic head and duodenum. The specimen was positive for GATA-3, p63, and CK20; trypsin, synaptophysin, chromogranin, CD56, CK7, BCL 10, and neuroendocrine markers were all negative. These findings in conjunction with the cellular histopathology were consistent with metastatic UC (Figure 4). Two of the five peripancreatic lymph nodes obtained were positive for carcinoma.

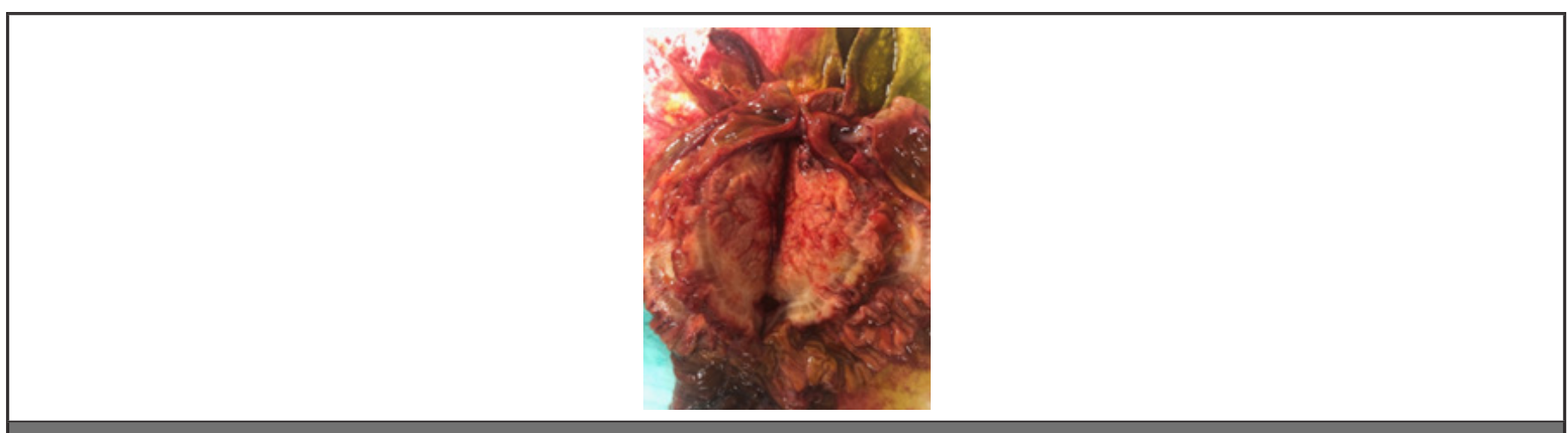

Figure 3: Gross specimen of urothelial carcinoma metastasis in the head of the pancreas causing desmoplastic reaction extending into the duodenum.
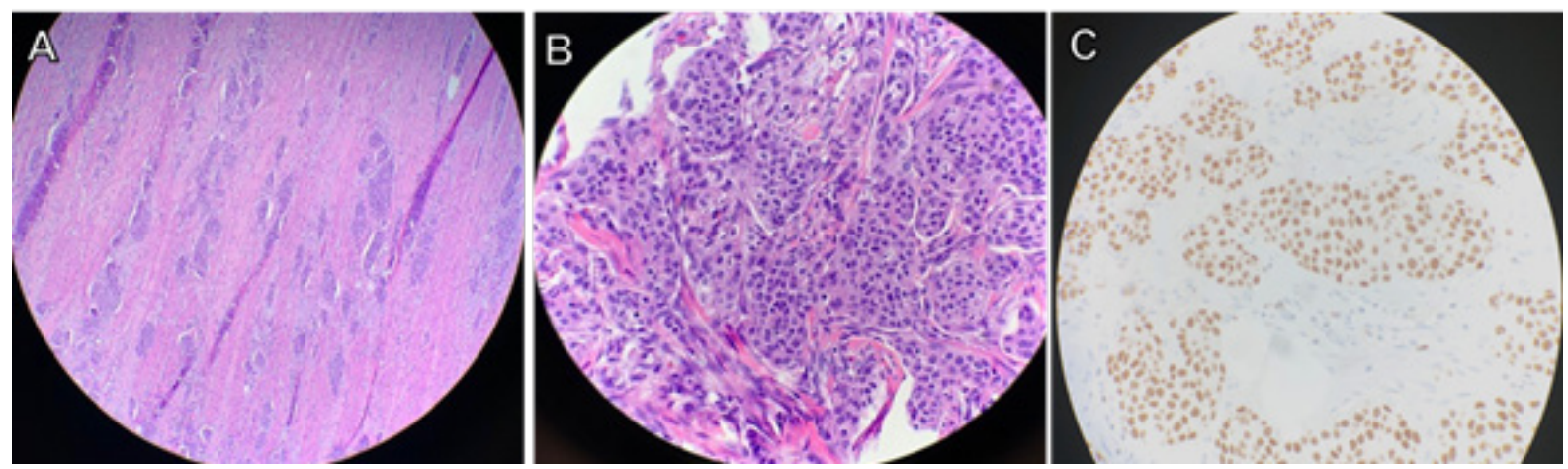

Figure 4: A) Metastatic urothelial carcinoma (x10 magnification) in the head of the pancreas and duodenum. B) Metastatic urothelial carcinoma ( $\times 40$ magnification) in the head of the pancreas and duodenum. C) GATA3 positive staining ( $\times 40$ magnification), indicative of metastatic urothelial carcinoma in the head of the pancreas and duodenum.

The patient's postoperative course was complicated by delayed gastric emptying requiring NGT re-insertion on several different occasions. On postoperative day 5 , a pancreatic leak was discovered requiring prolonged drainage via the drain left during surgery. Despite both mechanical and chemical prophylaxis, a deep venous thrombosis was found later in his postoperative course along with sub segmental pulmonary emboli. Long term anticoagulation was initiated and after tolerating adequate oral in take the patient was discharged with plans to start chemotherapy. 
The patient consulted with a urologist shortly after discharge. Repeat urine cytology was notable for hematuria and atypia. A cystoscopy was performed however there were no lesions within the bladder. Although positron emission tomography (PET scan) showed a hypermetabolic lesion in the mid-right ureter, the patient declined any further investigations to obtain selective cytology. Assuming upper tract UC as the primary, he was started on chemotherapy with a combination of Carboplatin and Gemcitabine. After several cycles, a repeat PET scan showed resolution of the hypermetabolic lesion.

Although the patient's condition remained stable for several months, his initial symptoms recurred with abdominal pain, nausea and vomiting. Subsequent work-up revealed a stricture at the gastrojejunal (G) anastomosis. This was initially managed with endoscopic dilation, however, after several weeks of relief, his symptoms recurred. A diagnosis of afferent loop syndrome was made, and he underwent a laparoscopic Braun's anastomosis. Findings on laparoscopy were notable for peritoneal nodules and desmoplastic reaction at the GJ anastomosis. Biopsies of these lesions later reported metastatic UC. With this unfortunate news, the patient elected to start hospice care.

\section{Discussion}

Bladder cancer is the most common urogenital tract (UGT) malignancy with UC as the predominant subtype [12]. It has a male predominance of 3:1 and is the 4th most common cancer among males in the United States [13].As was the case with our patient, painless hematuria is the most common presenting symptom, occurring in roughly $85 \%$ of patients [14]. While CT urography may be used to evaluate for local invasion, traditional CT imaging has a limited ability to detect bladder wall irregularities and other findings characteristic of urothelial cancer [15]. The gold standard for diagnosing UC is cystoscopy with biopsy. Once tissue has been obtained, immunohistochemistry (IHC) and histomorphology are utilized to differentiate UC from other cancers of the UGT. The IHC marker GATA3 is particularly useful as it has high sensitivity and specificity for both primary and metastatic UC $[16,17]$.

The prognosis of bladder cancer exists on a spectrum with 5-year survival rates ranging from $94 \%$ for localized disease to $2 \%$ for distant metastatic disease [18]. In broad terms, the staging of UC can be broken down into non-muscular invasive (i.e., T1 or less) and muscular invasive disease (i.e., T2a or greater) [19]. With muscular invasion, the prognosis of UC declines dramatically as the disease phenotype becomes much more aggressive. As such, the treatment of UC is highly dependent on whether or not muscular invasion is present. In patients without muscular invasion, transurethral resection of the bladder (TURB) with adjuvant chemotherapy is appropriate [20]. With muscular invasion, neoadjuvant chemotherapy followed by radical cystectomy (RC) is the standard treatment, after which the recurrence rate is approximately $30 \%$. In cases of recurrence, over $70 \%$ occur at distant sites beyond the pelvis [21]. Lymph nodes are the most common site of metastasis for UC and any involvement beyond the pelvis is indicative of stage IV disease [22].

Despite its ability to widely metastasize, UC metastasizing to the pancreas is an exceedingly rare event. Our review of the literature revealed only two other reports of such occurrences. In one case, metastatic disease was discovered while the patient was undergoing staging for bladder cancer [23]. The other case describes a presentation of pancreatitis and features concerning for rejection in a patient following pancreatic transplant [24]. The true diagnosis was then discovered upon biopsy of the pancreas allograft.

To our knowledge, this is the first reported case of duodenal obstruction caused by UC metastasizing to the pancreas. A broad range of pathologies can result in duodenal obstruction, from benign to malignant, intraluminal or extraluminal. Despite many benign etiologies, periampullary tumors remain the most common cause of duodenal obstruction [25]. As such, an adult presenting with duodenal obstruction has cancer until proven otherwise. While cancer was strongly suspected in this case, UC as the primary was highly unexpected. The majority of patients who present with periampullary obstruction will ultimately be diagnosed with pancreatic cancer (65\%), followed by bile duct (8\%), gastric (8\%), and ampullary cancer (7\%) [26]. Other causes such as metastatic disease is found in approximately $3 \%$ of patients. With the pancreas being the most common site for periampullary tumors, it is important to note that less than $40 \%$ of patients with pancreatic cancer will experience the nausea and vomiting classically associated with bowel obstruction [27]. More commonly patients present with symptoms of anorexia and abdominal pain. As these symptoms are vague, imaging is essential in the evaluation of such patients. CT is the modality of choice given its greater sensitivity and specificity compared to ultrasound or magnetic resonance imaging [28]. Regarding this case, there were indeed abnormal ureteral findings on CT imaging during the initial evaluation. However, these findings were thought to be incidental at the time given the patient's recent nephrolithiasis and history of ureteroscopy. Moreover, urogenital metastases causing duodenal obstruction is extraordinarily unusual.

In addition to rarity, this case was notable in that the diagnosis was made following pancreaticoduodenectomy (Whipple procedure). In terms of treatment for periampullary cancers, the 
Whipple procedure remains the cornerstone of management with curative intent. While this treatment was indicated for malignant duodenal obstruction without evidence of distant metastasis, surgery may have been deferred had metastatic UC been suspected. Although bladder resection is the primary treatment for UC, patients with distant metastases will likely benefit from neoadjuvant therapy [29]. In cases of unresectable disease, palliative options such as stenting or bypass of the obstruction may be considered. While there is evidence favoring a potential benefit from pancreatic metastasectomy, knowledge of oncologic outcomes has been limited by the paucity of this disease with contemporary evidence having been built primarily upon observational data [30]. With these limitations in mind, pancreatic metasectomy has been associated with improved overall survival and lower recurrence rates for select cancers [31]. However, as one may expect given its rarity, there is no literature available regarding oncologic outcomes following pancreatic metastasectomy for UC.

\section{Conclusion}

Although most patients presenting with duodenal obstruction will be diagnosed with cancer, metastatic disease is exceptionally rare. This is the first reported case of UC metastasizing to the pancreas presenting with duodenal obstruction. Pancreaticoduodenectomy remains the cornerstone of treatment with curative intent for select patients with localized periampullary cancer. While the primary treatment for UC is resection via radical cystectomy or TURB depending on the presence or absence of muscular invasion, respectively, the oncologic utility of pancreatic metastasectomy has yet to be determined.

\section{Conflict of Interest Statement}

The authors have no competing interests to declare. Informed consent was obtained from the patient presented. While no direct funding was received for the production of this work, this research was supported (in whole or in part) by HCA Healthcare and/or an HCA Healthcare affiliated entity. The views expressed in this publication represent those of the author(s) and do not necessarily represent the official views of HCA Healthcare or any of its affiliated entities.

\section{Reference}

1. Foroughi F, Mohsenifar Z, Ahmadvand A, Zare K (2012) Pathologic findings of Whipple pancreaticoduodenectomy: a 5-year review on 51 cases at Taleghani general hospital. Gastroenterol Hepatol Bed Bench 5(4): 179-182.

2. Yeo CJ, Cameron JL, Sohn TA, Lillemoe KD, Pitt HA, et al. (1997) Six Hundred Fifty Consecutive Pancreaticoduodenectomies in the 1990s: pathology, complications, and outcomes. Ann Surg 226(3): 248-257.

3. Smith AL, Odronic SI, Springer BS, Reynolds JP (2015) Solid Tumor Metastases to the Pancreas Diagnosed by FNA: A Single-Institution
Experience and Review of the Literature. Cancer Cytopathol 123(6): 347-355.

4. Bu K, Shi Z, Lu Y, Zhao J, Li B (2019) An occult urothelial carcinoma with wide multiorgan metastases and its genetic alteration profiling: Case report and literature review. Medicine (Baltimore) 98(16): e15245.

5. Lehmann J, Suttmann H, Albers P, Volkmer B, Gschwend JE, et al. (2009) Surgery for Metastatic Urothelial Carcinoma with Curative Intent: The German Experience. Eur urol 55(6): 1293-1299.

6. Shinagare AB, Ramaiya NH, Jagannathan JP, Fennessy FM, Taplin ME, et al. (2011) Metastatic Pattern of Bladder Cancer: Correlation with the characteristics of the primary tumor. AJR Am J Roentgenol 196(1): 117122.

7. Wymer KM, Antic T, Donnell PH (2016) Case Report of a Rare Presentation of Urothelial Carcinoma with Gastric Metastasis. Clin Genitourin Cancer 14(1): e111-114.

8. Weizer AZ, Shariat SF, Haddad JL, Escudier S, Lerner SP (2002) Metastatic transitional cell Carcinoma of the Urinary Bladder to the Shoulder Girdle. Rev Urol 4(2): 97-99.

9. Giordano G, Olivieri N, Andrea MR, Raimo TD, Manfrin E, et al. (2019) Urothelial bladder carcinoma metastasizing to the eye: A systematic review and case report. Oncol Lett 17(1): 462-467.

10. Jani N, Girotra M (2010) Bladder Cancer Metastasis to Duodenum: An Unusual Presentation of Obscure GI Bleed. Dig Dis Sci 55(6): 1801-1802.

11. Lees AN (2015) Cutaneous metastasis of transitional cell carcinoma of the urinary bladder eight years after the primary: a case report. J Med Case Rep 9: 102.

12. George M, Cumberbatch K, Jubber I, Black PC, Esperto F, et al. (2018) Epidemiology of Bladder Cancer: A Systematic Review and Contemporary Update of Risk Factors in 2018. Eur Urol 74(6): 784-795.

13. Martyn-hemphill C, Mak D, Khan MS, Challacombe BJ, Bishop CV (2013) Recent advances in diagnosis and treatment of transitional cell carcinoma of the bladder. Int J Surg 11(9): 749-752.

14. Kirkali Z, Chan T, Manoharan M, Algaba F, Busch C, et al. (2005) Bladder Cancer: Epidemiology, Staging and Grading, and Diagnosis. Urology 66(6 Suppl 1): 4-32.

15. Chaan S Ng (2006) Radiologic Diagnosis and Staging of Renal and Bladder Cancer. Semin Roentgenol 41(2): 121-138.

16. Chang A, Amin A, Gabrielson E, Illei P, Roden RB, et al. (2012) Utility of GATA3 Immunohistochemistry in Differentiating Urothelial Carcinoma From Prostate Adenocarcinoma and Squamous Cell Carcinomas of the Uterine Cervix, Anus, and Lung. Am J Surg Pathol 36(10): 1472-1476.

17. Zhao L, Antic T, Witten D, Paner GP, Taxy JB, et al. (2013) Is GATA3 Expression Maintained in Regional Metastases?: a study of paired primary and metastatic urothelial carcinomas Am J Surg Pathol 37(12): 1876-1881.

18. American Cancer Society (2019) Cancer Facts \& Figures. Atlata, USA.

19. Chou R, Buckley D, Fu R, Gore JL, Gustafson K, et al. (2015) Emerging Approaches to Diagnosis and Treatment of Non-Muscle-Invasive Bladder Cancer. Rockville (MD): Agency for Healthcare Research and Quality (US) Report No: 15(16)-EHC017-EF.

20. Burger M, Capoun O, Cohen D, Bohle A, Babjuk M, et al. (2017) EAU Guidelines on Non-Muscle-invasive Urothelial Carcinoma of the Bladder: Update 2016. Eur urol 71(3): 447-461.

21. Stein BJ, Lieskovsky G, Cote R, Groshen S, Feng AC, et al. (2001) Radical Cystectomy in the Treatment of Invasive Bladder Cancer: Long-Term Results in 1,054 Patients. J Clin Oncol 19(3): 666-675.

22. Campi R, Minervini A, Mari A, Hatzichristodoulou G, Sessa F, et al. (2017) Anatomical Templates of Lymph Node Dissection for Upper Tract 
Urothelial Carcinoma-a Systematic Review of the Literature. Expert Rev Anticancer Ther 17(3): 235-246

23. Canter D, Simhan J, Smaldone MC, Labed B, Tokar JL, et al (2012) Clinical Stage T1 Micropapillary Urothelial Carcinoma Presenting With Metastasis to the Pancreas. Urology 79(2) :e9-10.

24. Wang J, Talmon G, Jaffar SA, Siref LE, Morris MC (2012) Metastases From Nested Variant Urothelial Carcinoma of the Urinary Bladder in Pancreatic Allograft Mimicking Graft Rejection. J Clin Med Res 4(2): 145148.

25. Sarani B, Paspulati R, Hambley J, Efron D, Martinez J, et al. (2018) A multidisciplinary approach to diagnosis and management of bowel obstruction. Curr Probl Surg 55(10): 394-438.

26. Hamada T, Nakai Y, Lau JY, Moon JH, Hayashi T, et al. (2018) Internationa study of endoscopic management of distal malignant biliary obstruction combined with duodenal obstruction. Scand J Gastroenterol 53(1): 4655.

27. Porta M, Fabregat X, Malats N, Guarner L, Carrato A, et al. (2005) Exocrine Pancreatic Cancer: Symptoms at Presentation and Their Relation to Tumour Site and Stage. Clin Transl Oncol 7(5): 189-197.
28. Bipat S, Phoa SS, Delden OM, Bossuyt PM, Gouma DJ, et al. (2005) Ultrasonography, Computed Tomography and Magnetic Resonance Imaging for Diagnosis and Determining Resectability of Pancreatic Adenocarcinoma: a Meta-analysis. J Comput Assist Tomogr 29(4): 438445

29. Grossman HB, Natale RB, Tangen CM, Speights VO, Vogelzang NJ, et al. (2003) Neoadjuvant Chemotherapy plus Cystectomy Compared with Cystectomy Alone for Locally Advanced Bladder Cancer. N Engl J Med 349(9): 859-866

30. Madkhali AA, Shin S, Song KB, Lee JH, Hwang DW, et al. (2018) Pancreatectomy for a secondary metastasis to the pancreas : A singleinstitution experience. Medicine (Baltimore) 97(42): e12653.

31. Adler H, Redmond CE, Heneghan HM, Swan N, Maguire D, et al. (2014) Pancreatectomy for metastatic disease: A systematic review. Eur J Surg Oncol 40(4): 379-386. 